\title{
Binding energies of positive and negative trions: From quantum wells to quantum dots
}

\author{
A. S. Bracker, E. A. Stinaff, D. Gammon, M. E. Ware, J. G. Tischler, and D. Park \\ Naval Research Laboratory, Washington, DC 20375, USA \\ D. Gershoni \\ Physics Department, Technion-Israel Institute of Technology, Haifa, 32000, Israel \\ A. V. Filinov and M. Bonitz \\ Christian-Albrechts-Universität zu Kiel, Institut für Theoretische Physik und Astrophysik, Leibnizstrasse 15, 24098 Kiel, Germany \\ F. Peeters and C. Riva \\ Departement Fysica, Universiteit Antwerpen (Drie Eiken Campus), Universiteitsplein 1, B-2610 Antwerpen, Belgium
}

(Received 21 August 2004; published 15 July 2005)

\begin{abstract}
We compare binding energies for positive and negative trions in a series of narrow GaAs quantum wells and in "natural" quantum dots defined by quantum well thickness fluctuations. We assign photoluminescence features to oppositely charged trions through a combination of charging behavior, luminescence polarization, and spin fine structure. Negative trions are found to have a higher binding energy than positive trions. Our observations compare well with path integral Monte Carlo calculations for different well widths. This comparison provides a physical interpretation of the observed trends and sheds light on a longstanding disagreement between theory and experiment on the influence of lateral confinement on trion binding energies.
\end{abstract}

DOI: 10.1103/PhysRevB.72.035332

PACS number(s): 78.67.De, 78.67.Hc, 71.35.Pq, 72.25.Fe

\section{INTRODUCTION}

The binding energies of electron-hole complexes in quantum wells (QWs) are higher than bulk values, because of the enhanced Coulomb interaction between confined carriers. Charged excitons (trions) were first observed in II-VI QWs (Ref. 1) and shortly thereafter in GaAs-AlGaAs QWs. ${ }^{2-4}$ Not only were the measured binding energies higher than bulk values, but they also exceeded most theoretical estimates of trion binding energies in QWs (see Ref. 5 for a recent review). At that time, the theory considered perfect QWs, and the influence of remote donor ions or interface thickness fluctuations was not included. However, two-dimensional imaging experiments have shown that donor ions produce a corrugated potential that localizes trions even in wide wells, ${ }^{6}$ and it is well known that interface roughness in narrow wells can confine carriers in quantum dot-like potentials. In the past few years, theoretical work has supported the idea that binding energies are enhanced by lateral confinement potentials. $^{7,8}$

The difference between binding energies for positive $\left(X^{+}\right)$ and negative $\left(X^{-}\right)$trions in GaAs QWs also remains unresolved. In a narrow II-VI QW, $X^{+}$has been shown to have a smaller binding energy. ${ }^{9}$ In wider GaAs QWs $(>20 \mathrm{~nm})$, the binding energies were observed to be the same, ${ }^{10-12}$ except in the presence of a magnetic field. ${ }^{10,11}$ Recent calculations ${ }^{7,8}$ predict that binding energies may differ strongly in narrow QWs due to the influence of lateral confinement, although so far this has not been demonstrated experimentally.

In the present work we report experimental results that address the influence of lateral confinement potentials and differences between oppositely charged trions, and we compare the results with theory. In order to establish a clear assignment of positive and negative trions, we begin with trions confined in individual "natural" quantum dots (QDs) that are defined by the large monolayer-height islands at the interfaces of a narrow QW. The simple photoluminescence (PL) spectra of individual QDs reveal clear signatures that distinguish $X^{+}$from $X^{-}$. By changing the experimental conditions, we can study $X^{+}$or $X^{-}$within the same sample. We have measured trion binding energies for a few dozen such QDs in different types of samples. Having identified the characteristic behavior of $X^{+}$and $X^{-}$in individual QDs, we then consider ensemble PL spectra for QWs of several different widths. We can again study both $X^{+}$and $X^{-}$within the same sample and obtain approximate binding energies from partially-resolved exciton and trion peaks that are inhomogeneously broadened by the distribution of interface potentials. We compare the ensemble results with the single QD results and with path integral Monte Carlo calculations that consider the influence of interface thickness variations on trion binding energies.

\section{EXPERIMENT}

Two types of $\mathrm{GaAs}-\mathrm{Al}_{0.3} \mathrm{Ga}_{0.7} \mathrm{As}$ quantum well samples were investigated. All samples were grown with two minute growth interrupts at the QW interfaces, which leads to the formation of large monolayer (ML) thickness variations. The first type of sample consisted of five modulation-doped QWs ( $p$-type or $n$-type, $3 \times 10^{10} \mathrm{~cm}^{-2}$ ), with nominal widths of $14.1 \mathrm{~nm}$ (50 ML), $8.5 \mathrm{~nm}$ (30 ML), $6.2 \mathrm{~nm}$ (22 ML), $4.2 \mathrm{~nm}$ $(15 \mathrm{ML})$, and $2.8 \mathrm{~nm}(10 \mathrm{ML})$. Further details on these types of sample are provided in Ref. 13. The second type of sample was a single quantum well embedded in an $n-I$ ( $p$ I) Schottky diode. The heterostructure was grown on an $n^{+}$ 
$\left(p^{+}\right)$-doped substrate with a $1 \mu \mathrm{m} \mathrm{GaAs}$ buffer layer doped with silicon (beryllium) at $5 \times 10^{17} \mathrm{~cm}^{-3}$. The heterostructure consisted of $20 \mathrm{~nm} \mathrm{Al} \mathrm{Al}_{0.3} \mathrm{Ga}_{0.7}$ As $\left[2.5 \times 10^{17} \mathrm{~cm}^{-3} \mathrm{Si}(\mathrm{Be})\right]$, $80 \mathrm{~nm}$ undoped AlGaAs, a GaAs quantum well (nominally 2.8 or $4.2 \mathrm{~nm}$ ), a $100 \mathrm{~nm} \mathrm{AlGaAs} \mathrm{top} \mathrm{barrier} \mathrm{and} \mathrm{a} 10 \mathrm{~nm}$ GaAs capping layer. A semitransparent titanium electrode ( $\sim 8 \mathrm{~nm}$ ) was evaporated on the samples. For single QD spectroscopy, aluminum masks with submicron apertures were fabricated by electron beam lithography and lift-off techniques. Electrical contacts were made to the top gate and to the substrate with low temperature solder, silver epoxy, and/or wire bonding. The samples were mounted in standard cryostats and cooled to temperatures between $5 \mathrm{~K}$ and $8 \mathrm{~K}$. They were excited with a Ti:sapphire laser tuned into the quasicontinuum above the lateral QD potential barrier. PL spectra were resolved in a 1.5 meter triple spectrometer and detected with a multichannel CCD detector.

\section{PHOTOLUMINESCENCE SPECTRA}

The diode heterostructures allow a rapid preliminary assignment of $X^{\circ}, X^{+}$, and $X^{-}$based on changes in the PL spectrum when an individual QD becomes charged with an electron or hole. Figure 1(a) shows how a typical QD PL spectrum changes as a function of the applied bias of a $p-I$ diode. We see a single strong peak at zero bias, and when the bias is raised to $0.5 \mathrm{~V}$, there is a discrete shift of the peak to higher energy. We assign the peaks to $X^{+}$and $X^{\circ}$, with the transition at $0.5 \mathrm{~V}$ corresponding to expulsion of a hole from the QD. The peak separation of roughly $2.5 \mathrm{meV}$ corresponds to the trion binding energy. Biexciton peaks $X X^{\circ}$ and $X X^{+}$are observed in the spectrum at higher laser intensities.

A typical spectrum for a QD in an $n-I$ diode is shown in Fig. 1(b). Here, three main peaks are observed. The transition at $+1.2 \mathrm{~V}$ corresponds to injection of an electron into the QD, producing $X^{-} .{ }^{14}$ At lower biases where $X^{\circ}$ exists, we often observe $X^{+}$. In our $n-I$ diodes, $X^{+}$may be produced if a photoelectron tunnels out of the quantum well, leaving an excess hole. We have not observed $X^{-}$in $p-I$ diodes, possibly due to slower tunneling times for holes. The production of oppositely-charged trions within a single quantum well has been reported previously. ${ }^{9-11}$

The circular polarization of the PL supports the assignment of $X^{\circ}, X^{+}$, and $X^{-}$. The details of spin polarization in natural QDs are discussed in a separate publication. ${ }^{15}$ Briefly, the $X^{+}$polarization is governed by the spin of an unpaired photoelectron, so that its PL polarization is large and positive, i.e., with the same helicity as the laser. The $X^{\circ}$ shows smaller PL polarization (sometimes zero), because its spin can be depolarized by the anisotropic electron-hole exchange interaction. ${ }^{16}$ The polarization of $X^{-}$can take either positive or negative values depending on laser power, excitation energy, and applied bias. This complex behavior is related to optical pumping of electrons and to the dynamics of trion formation. ${ }^{15}$ Our assignment of trions in QW ensemble spectra will make use of the tendency for $X^{+}$and $X^{-}$to have positive and negative polarization, respectively.

Modulation-doped samples can also contain either positive or negative net charge in a QW under different experi-
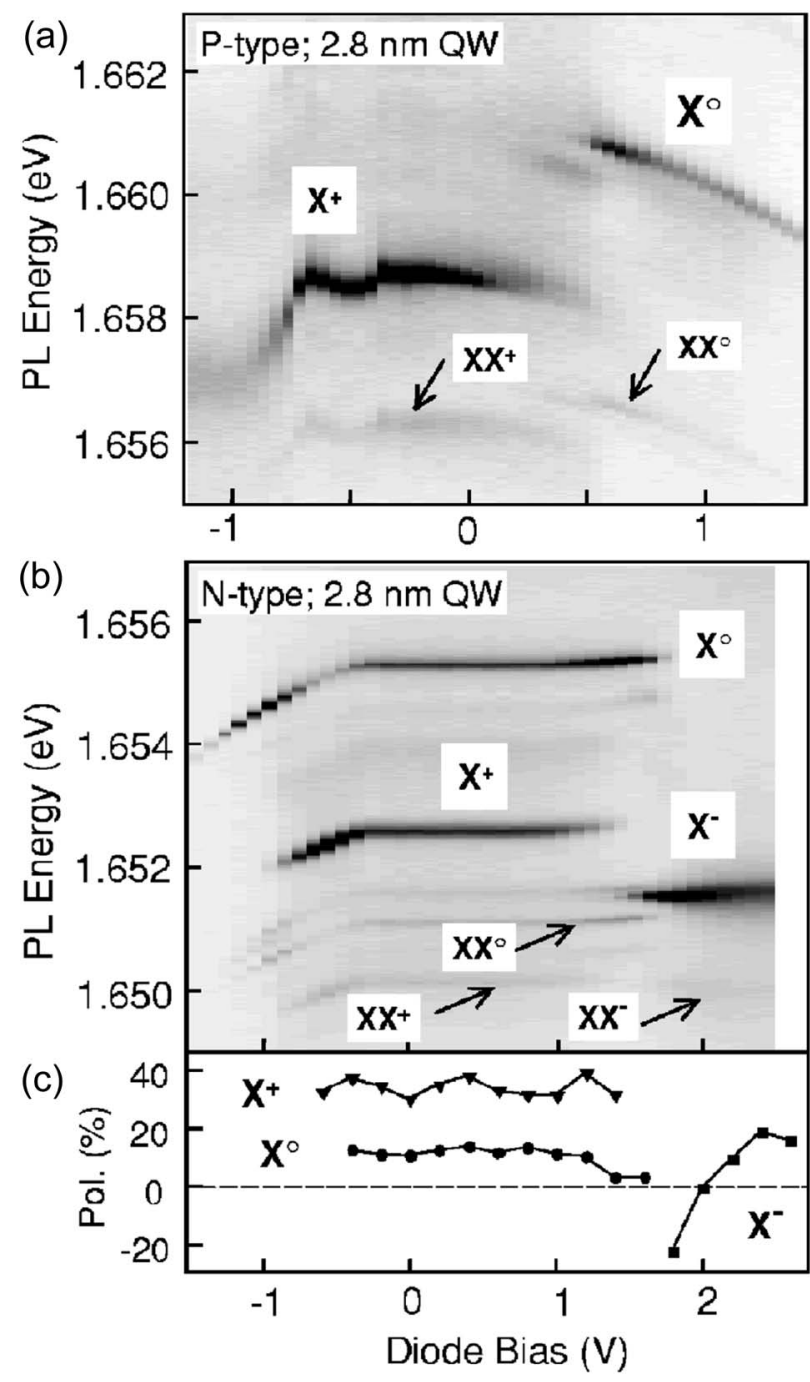

FIG. 1. (a) Photoluminescence intensity (gray scale) for a single natural quantum dot in a $2.8 \mathrm{~nm}$ (nominal width) GaAs quantum well embedded in a $p$-type Schottky diode, as a function of emitted photon energy and applied bias. Neutral exciton $\left(X^{\circ}\right)$, positive trion $\left(X^{+}\right)$, and biexcitons $\left(X X^{\circ}, X X^{+}, X X^{-}\right)$are labeled. (b) Analogous spectrum for an $n$-type Schottky diode, which shows the negative trion $\left(X^{-}\right)$. (c) Photoluminescence circular polarization as a function of bias for the QD shown in (b).

mental conditions. A striking example is shown in Fig. 2(a) for an individual natural quantum dot within an $n$-type modulation-doped $4.2 \mathrm{~nm} \mathrm{QW}$. Small changes in the laser energy produce sudden shifts in the PL peak energy. These shifts are observed for most of the other QD PL peaks in the spectrum (not shown here; see Ref. 17), suggesting that the change is not a resonance of the individual QD, but a property of the QW. The pattern of spacings for the three main PL peaks in Fig. 2(a) is very similar to that observed in the diode samples, suggesting that we are again observing $X^{\circ}, X^{+}$, and $X^{-}$. Furthermore, the PL polarization ${ }^{17}$ agrees qualitatively with the observations in the diode sample.

Spin fine structure confirms our assignment of the neutral exciton and trions. ${ }^{13}$ In the presence of a magnetic field in the Voigt geometry (field vector in the plane of the quantum well), a second peak develops in the $X^{\circ}$ spectrum, shifted to 


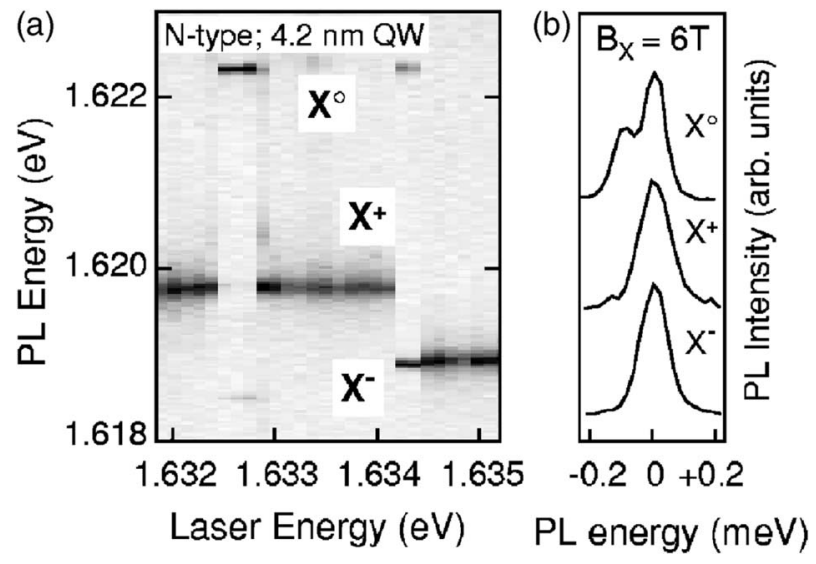

FIG. 2. (a) Photoluminescence intensity (gray scale) for a single natural quantum dot in a $4.2 \mathrm{~nm}$ (nominal width) $n$-type modulation-doped GaAs quantum well, as a function of emitted photon (PL) energy and laser excitation energy. (b) All three peaks from the same QD in a $6 \mathrm{~T}$ magnetic field (Voigt geometry). $X^{\circ}$ exhibits exchange fine structure.

lower energy by roughly $100 \mu \mathrm{eV}$. This corresponds to a "forbidden" transition that becomes allowed in the magnetic field, and the peak spacing corresponds to the exchange interaction between unpaired electron and hole spins. There is no exchange fine structure for $X^{-}\left(X^{+}\right)$because the electrons (holes) are paired in a singlet configuration. Notably, $X^{-}$and $X^{+}$show the same behavior. In an earlier investigation of exciton and trion fine structure, ${ }^{13}$ we identified a trion PL peak as $X^{-}$. However with the benefit of more recent data on binding energies, we recognize that the QDs investigated in that work were in fact positively charged.

We next consider trions and excitons observed in ensemble PL spectra for GaAs quantum wells of different widths. Our spectra simultaneously show features from neutral excitons and trions. Previous work (see, e.g., Ref. 6) has shown that this behavior is typical of photoluminescence spectra for wide QWs. This contrasts to the broad electron gas feature that dominates the PL spectrum when the electron concentration is higher. Our spectra are inhomogeneously broadened by a distribution of lateral confinement potentials. The single QD spectra discussed so far were obtained from the low energy wings of the ensemble spectra for the two narrowest quantum wells, and we find that the binding energies obtained from the ensemble spectra are considerably less than those of the corresponding QDs. This fact allows us to make a connection between earlier studies of trion binding energies in QWs and the atypical interface potentials investigated in our single QD work.

Ensemble PL spectra for four of the five QWs in modulation doped samples are shown in Fig. 3. All of the spectra come from one $p$-type sample, with the exception of the top solid-line spectrum, which comes from an $n$-type sample. Solid and dotted lines for each QW correspond to two different laser energies. The top two pairs of ensemble spectra ( $14.1 \mathrm{~nm}$ and $8.5 \mathrm{~nm}$ ) show four partially-resolved peaks each, corresponding to exciton and trion from two predominant well widths that differ by one monolayer. The two lower pairs of ensemble spectra $(6.2 \mathrm{~nm}$ and $4.2 \mathrm{~nm})$ each show

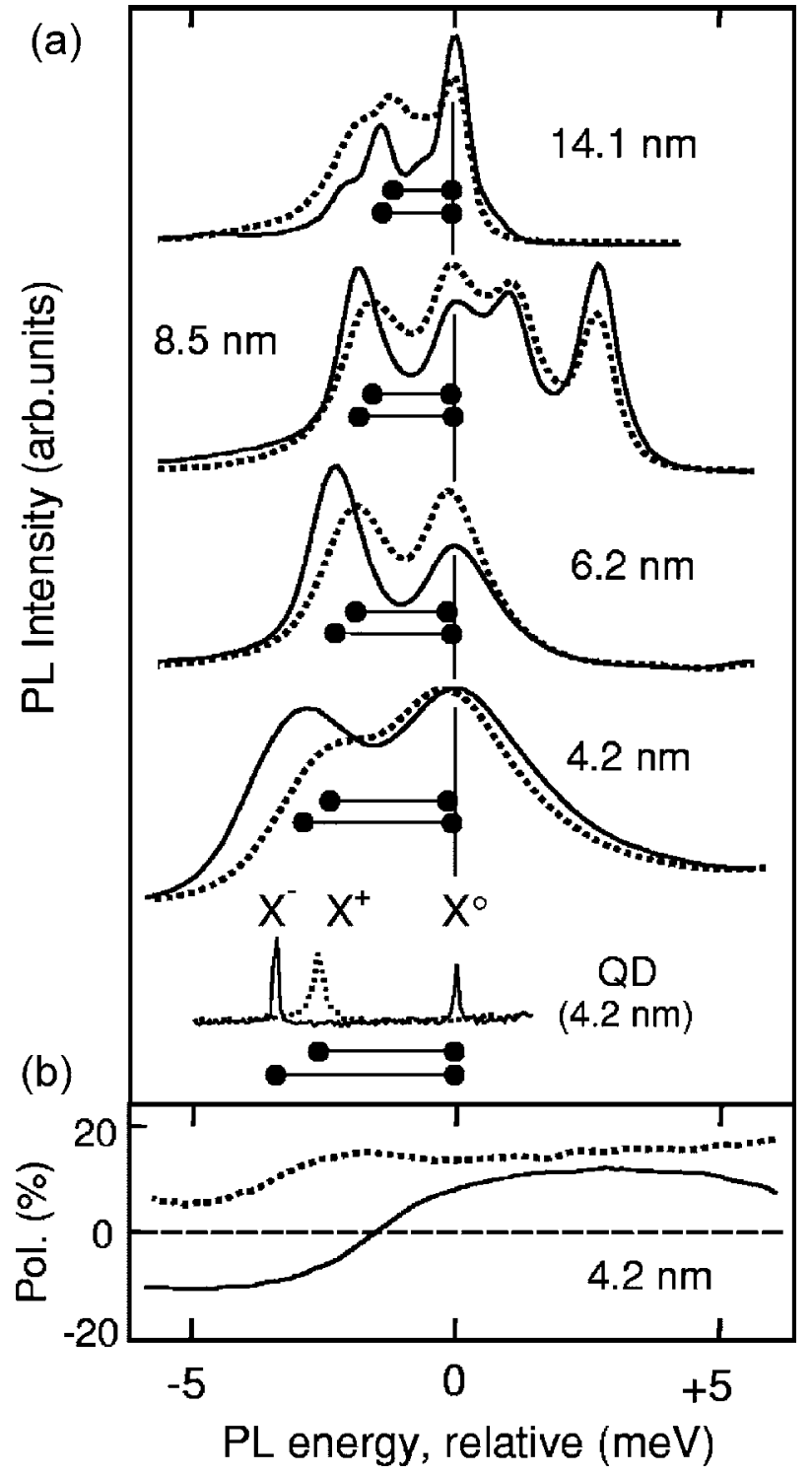

FIG. 3. (a) Ensemble photoluminescence spectra for GaAs quantum wells with four different widths. The top two pairs of spectra correspond to two well widths each, differing by one monolayer (see text). One single QD is shown for comparison. Solid and dotted lines correspond to different laser excitation energies, chosen separately for each well. Horizontal bars span exciton-trion splittings for each spectrum. (b) Photoluminescence circular polarization for the $4.2 \mathrm{~nm}$ ensemble spectra.

two peaks, corresponding to excitons and trions within a single monolayer. For these narrower wells, the upper monolayer features are observed at higher energy, out of range on the scale shown. We do not show spectra from the narrowest $(2.8 \mathrm{~nm}) \mathrm{QW}$, where the exciton and trion peaks are not resolved.

The distinctive trion peak shifts that are observed for the single QD spectra in Fig. 2(a) are also observed in the ensemble, by comparing the spectra with solid and dotted lines, which correspond to two different laser energies. We find that the trion peak shifts quasidiscretely as the laser energy is tuned, while the exciton peak moves very little. Unlike in the single QD spectrum, excitons and trions are observed simul- 
taneously with only their relative intensities changing as the laser energy is varied. We argue that the PL energy shift of the ensemble trion peak corresponds to a change between positive and negative trions. This assignment is supported in Fig. 3(b) by polarization data for the $4.2 \mathrm{~nm}$ QW. The trion features with larger and smaller exciton-trion splittings have negative and positive PL polarization, respectively. The situation with the ensemble therefore appears very similar to the single QD spectra, with the negative trion having the larger binding energy.

\section{BINDING ENERGIES}

In order to obtain binding energies from the QW ensemble PL spectra, we measured the characteristic energy separation between the exciton and trion peaks. The spectra were fit with Gaussian functions to approximate the shape of individual peaks in an inhomogeneously broadened spectrum. Spectra were obtained over a wide range of excitation energies, two of which are shown for each QW in Fig. 3. From the subset of spectra that showed large intensities for both trion and exciton, we selected those with the largest and smallest values of the exciton-trion splitting.

For single quantum dots, we measured the exciton-trion splitting for a few dozen quantum dots in four different samples, including $n$-type and $p$-type Schottky diode QWs and modulation-doped QWs. The results of these individual measurements are shown in the inset to Fig. 4 as a function of the PL energy of the exciton peak. Two nominal quantum well widths, $2.8 \mathrm{~nm}$ and $4.2 \mathrm{~nm}$, are represented.

Measured binding energies from ensemble PL spectra (solid symbols) and individual QDs (hollow symbols) are shown in the main part of Fig. 4 as a function of the quantum well width. The QD points represent the average of the individual measurements shown in the inset. PL energies were converted into well width units using a calibration curve generated from the exciton peak positions for several samples containing five $\mathrm{GaAs}-\mathrm{Al}_{0.3} \mathrm{Ga}_{0.7}$ As quantum wells of known width. The calibration curve was corrected for the Stokes shift, which produces the most intense PL peak for a QW that is one monolayer thicker than the nominal (intended) QW width. For the single QD points, two additional effects cause the measured well widths to exceed their corresponding nominal well widths of $2.8 \mathrm{~nm}$ and $4.2 \mathrm{~nm}$. First, the single QD spectra were taken from the low energy wings of the QW spectra, corresponding to a wider region of the well. Furthermore, the GaAs growth rate was slightly larger than intended. Using the calibration curve, we accounted for both of these effects in Fig. 4.

The binding energy measurements shown in Fig. 4 reveal three distinctive trends. In addition to the well-established increase for narrow wells, we observe substantial differences between positive and negative trions and between single QDs and QW ensembles. Binding energies for negative trions exceed those for positive trions for all of the well widths considered here, with the largest fractional difference for the narrowest wells: 45\% larger for individual QDs and 33\% larger for QWs. Furthermore, QDs show larger binding energies than QWs, e.g., $30 \%$ larger for $X^{-}$and $17 \%$ larger for $X^{+}$in the narrowest well.

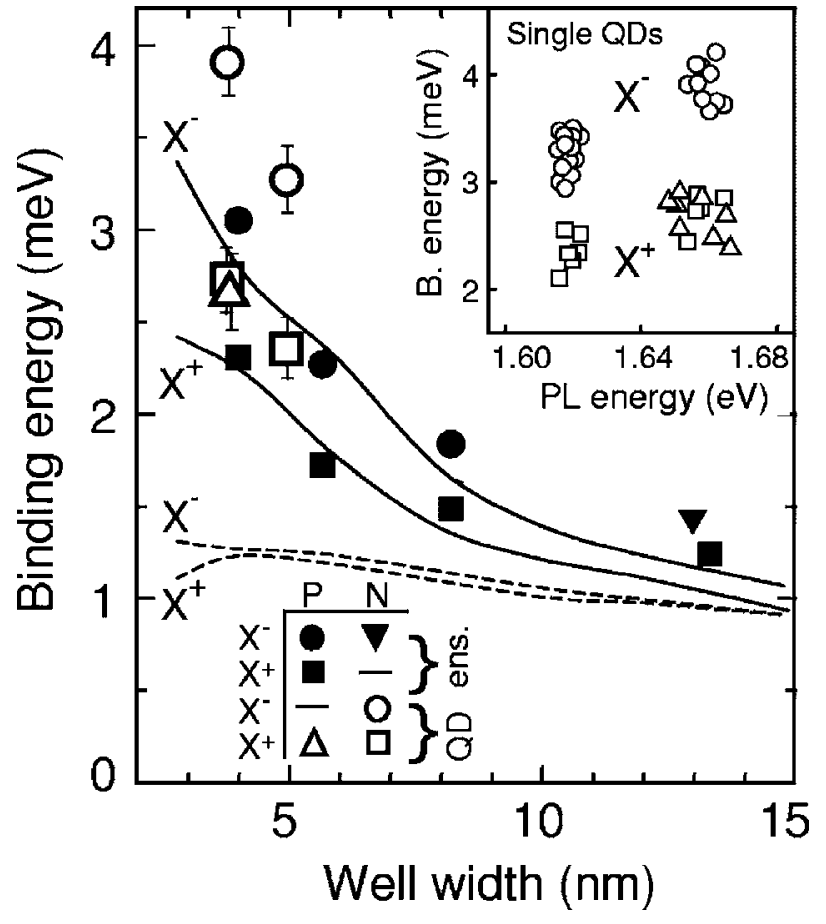

FIG. 4. Binding energies obtained from PL spectra of quantum well ensembles (solid symbols) and individual natural quantum dots (open symbols). Symbol shapes for $X^{-}$vs $X^{+}$, ensemble vs quantum dots, and $p$-type vs $n$-type samples are specified in the legend. Lines show PIMC calculations for $X^{-}$and $X^{+}$in quantum wells with interface fluctuations (diameter $D=30 \mathrm{~nm}$, solid lines) and perfectly smooth interfaces $(D=\infty$, dashed lines). The inset shows experimental results for the individual quantum dots that were averaged and converted to well width units to obtain the corresponding open symbols in the main graph.

Path integral Monte Carlo (PIMC) calculations of trion binding energies are shown in Fig. 4 (solid and dashed lines). The dashed lines are PIMC results for QWs with perfectly smooth interfaces (ideal QWs), while the solid lines are for trions laterally-confined in interface thickness fluctuation defects (QDs) with $30 \mathrm{~nm}$ diameter $(D)$ and 1 ML thickness. We used the Hamiltonian discussed in Ref. 8 with the following parameters: $V_{e}=0.57\left(1.155 x+0.37 x^{2}\right) \mathrm{eV}$ and $V_{h}$ $=0.43\left(1.155 x+0.37 x^{2}\right) \mathrm{eV}$, which are the height of the electron and hole square well confining potentials, respectively. Other parameters (also in Ref. 8) are Al concentration $x$ $=0.3$, dielectric constant 12.58, electron mass $m_{e}=0.067 m_{o}$, hole in-plane mass $m_{h}{ }^{\|}=0.112 m_{o}$, and hole mass in the QW growth direction $m_{h}^{z}=0.377 m_{o}$. Interface fluctuations are simulated through a cylindrically symmetric potential with a lateral diameter $D$ and depth $V_{e(h)}$ loc. The defect diameter was varied in the range $10 \mathrm{~nm}<D<80 \mathrm{~nm}$. The present model is reliable for well widths in the range $3 \mathrm{~nm}<L$ $<12 \mathrm{~nm}$. Beyond this region, full 3D calculations for the binding energies would be required.

The depth of the localization potential was obtained as the difference between the lowest energy levels of the electron (hole) in two QWs with the thickness differing by a single monolayer. Because of the different masses of the electron and hole, the depths of their localization potentials are dif- 


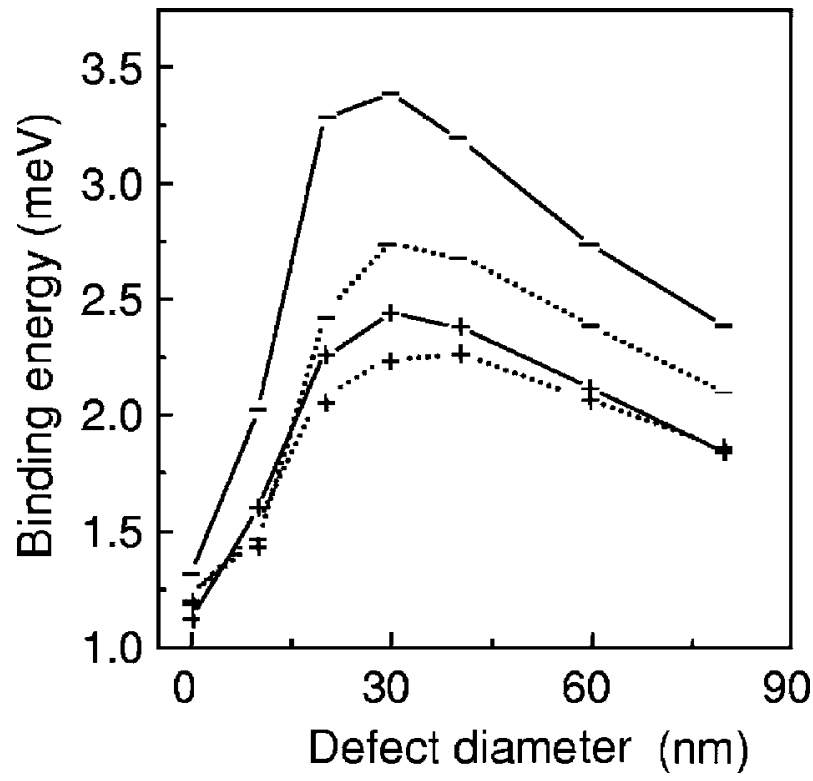

FIG. 5. PIMC calculations of the binding energy of negative and positive trions (symbols - and + , respectively) vs diameter of a 1 ML width fluctuation for a QW width $L=2.8 \mathrm{~nm}$ (solid lines) and $4.2 \mathrm{~nm}$ (dotted lines).

ferent. For example, for the QW width $L=4.2 \mathrm{~nm}$, the electrons feel a lateral confinement with depth $V_{e}^{\text {loc }}=5.93 \mathrm{meV}$, while for the holes, due to their larger mass, the confinement depth is $V_{h}^{\text {loc }}=2.61 \mathrm{meV}$. The depth of the localization potential decreases as the $\mathrm{QW}$ width increases, e.g., for $L$ $=8 \mathrm{~nm}$ we found that the confinement depths are only $V_{e}^{\text {loc }}$ $=2.04 \mathrm{meV}$ and $V_{h}^{\text {loc }}=0.61 \mathrm{meV}$. More details about the simulation and the parameters that were used can be found in Ref. 8.

\section{DISCUSSION}

The PIMC calculations for laterally confined trions reproduce the experimental trend that the binding energies are larger for $X^{-}$than for $X^{+}$. This effect decreases as the well width increases, because the lateral confinement caused by interface fluctuations is reduced. As discussed in Ref. 8, the lateral confinement carries two contributions to the binding energies. One contribution comes from the smaller average electron-hole distance in $X^{-}$compared to $X^{+}$, which enhances the $e-h$ Coulomb attraction. The other contribution is a larger gain in lateral localization energy for an electron compared to a hole, due to the smaller effective mass or likewise the larger mobility of the electron.

The calculations with interface fluctuations show better agreement with experiment than do the calculations for smooth interfaces. This is consistent with the known interface morphology of QWs grown with interrupts at the interfaces. Such QWs typically have interface fluctuations with lateral size ranging from $10-200 \mathrm{~nm},{ }^{16}$ and the ensemble spectra are characteristic of this distribution. The theoretical curves nicely reproduce the experimental results, although the agreement is not as close for well widths $L>12 \mathrm{~nm}$. A possible origin of this discrepancy is the adiabatic approximation - separation of particle motion in the QW plane and in the growth direction-used in the present calculations (see Ref. 8). However, for narrow QWs, where there is good agreement with the experiment, use of the adiabatic approximation is well grounded.

Another interesting feature that can be seen from the comparison in Fig. 4 is the unusually high trion binding energies in individual natural QDs. For example, for $X^{-}$in the QWs with widths $L=3.7 \mathrm{~nm}$ and $4.8 \mathrm{~nm}$ (nominal QW widths $L$ $=2.8 \mathrm{~nm}$ and $4.2 \mathrm{~nm}$, respectively), the experiment gives $E_{B}{ }^{\text {exp }}=3.9 \mathrm{meV}$ and $3.3 \mathrm{meV}$. These larger binding energies are not typical representatives of the ensemble. In general, this is not surprising, as the single QD spectra were taken from the low energy wing of the ensemble spectrum, where individual sharp PL peaks were well separated. The theoretical results for the well widths $L=3.68 \mathrm{~nm}$ and $4.76 \mathrm{~nm}$ and typical defect diameters in the range $D=30-40 \mathrm{~nm}$ give lower values $E_{B}{ }^{\text {theor }}=2.9 \pm 0.1 \mathrm{meV}$ and $2.6 \pm 0.1 \mathrm{meV}$.

In order to better understand the results for individual QDs, we have investigated the dependence of the trion binding energy on the defect diameter $D$. The results are shown in Fig. 5 for both $X^{-}$and $X^{+}$. A pair of curves corresponds to the nominal QW widths $L=2.8 \mathrm{~nm}$ and $4.2 \mathrm{~nm}$ plus a $1 \mathrm{ML}$ fluctuation defect. One can see that for both trions, a maximum for $E_{B}$ is reached around $D=30 \mathrm{~nm}(L=4.2 \mathrm{~nm})$ and takes the value $E_{B}=2.75 \mathrm{meV}$ for $X^{-}$and $E_{B}=2.23 \mathrm{meV}$ for $X^{+}$. This is related to the fact (shown in Figs. 4 and 6 of Ref. 8 ) that both the average interparticle distance and the gain in localization energy as a function of the defect diameter reach a maximum around $D=30 \mathrm{~nm}$. These values place an upper bound on the binding energy (for a given $L$ ) that can be obtained in the framework of the present localization model ${ }^{8}$ of a cylindrical defect of $1 \mathrm{ML}$ depth. This bound is still below the experimental results, leaving open the question about the high values of the binding energy in individual QDs. Possible sources for these high values are the anisotropic shape of the defects or the presence of nearby charged impurities. Both situations would require additional refinement of the calculations.

In summary, the trends in our experimental data on binding energies of positive and negative trions in quantum wells are clearly reproduced by numerical simulations, in which we assume that trions are localized on monolayer well width fluctuations. Lateral localization is responsible for the strong increase in trion binding energies as the well width decreases. The theory shows clearly that for localized trions, the negative trion $X^{-}$has a markedly higher binding energy than its positive counterpart $X^{+}$. Good agreement between the ensemble results and the calculations suggests that the main contribution to our quantum well photoluminescence spectra comes from localized states.

\section{ACKNOWLEDGMENTS}

This work was supported by DARPA, ONR, NSA/ARDA, and the Belgian Science Policy and the European Commission GROWTH Programme NANOMAT Project, Contract No. G5RD-CT-2001-00545. E.A.S. and M.E.W. were supported by NRC-NRL. C.R. is supported by FWOVlaanderen. 
${ }^{1}$ K. Kheng, R. T. Cox, Y. Merle d'Aubigné, F. Bassani, K. Saminadayar, and S. Tatarenko, Phys. Rev. Lett. 71, 1752 (1993).

${ }^{2}$ G. Finkelstein, H. Shtrikman, and Israel Bar-Joseph, Phys. Rev. Lett. 74, 976 (1995).

${ }^{3}$ A. J. Shields, M. Pepper, D. A. Ritchie, M. Y. Simmons, G. A. C. Jones, Phys. Rev. B 51, 18049 (1995).

${ }^{4}$ H. Buhmann, L. Mansouri, J. Wang, P. H. Beton, N. Mori, L. Eaves, M. Henini, and M. Potemski, Phys. Rev. B 51, 7969 (1995).

${ }^{5}$ F. M. Peeters, C. Riva, and K. Varga, Physica B 300, 139 (2001).

${ }^{6}$ G. Eytan, Y. Yayon, M. Rappaport, H. Shtrikman, and I. BarJoseph, Phys. Rev. Lett. 81, 1666 (1998).

${ }^{7}$ L. C. O. Dacal, R. Ferreira, G. Bastard, and J. A. Brum, Phys. Rev. B 65, 115325 (2002).

${ }^{8}$ A. V. Filinov, C. Riva, F. M. Peeters, Yu. E. Lozovik, and M. Bonitz, Phys. Rev. B 70, 035323 (2004).

${ }^{9}$ G. V. Astakhov, D. R. Yakovlev, V. P. Kochereshko, W. Ossau, W. Faschinger, J. Puls, F. Henneberger, S. A. Crooker, Q. McCulloch, D. Wolverson, N. A. Gippius, and A. Waag, Phys. Rev. B 65, 165335 (2002).

${ }^{10}$ S. Glasberg, G. Finkelstein, H. Shtrikman, and I. Bar-Joseph,
Phys. Rev. B 59, R10425 (1999); C. Riva, F. M. Peeters, and K. Varga, Phys. Rev. B 64, 235301 (2001).

${ }^{11}$ O. V. Volkov, V. E. Zhitomirskii, I. V. Kukushkin, K. von Klitzing, and K. Eberl, JETP Lett. 67, 744 (1998).

${ }^{12}$ A. J. Shields, J. L. Osborne, M. Y. Simmons, M. Pepper, and D. A. Ritchie, Phys. Rev. B 52, R5523 (1995).

${ }^{13}$ J. G. Tischler, A. S. Bracker, D. Gammon, and D. Park, Phys. Rev. B 66, 081310(R) (2002).

${ }^{14}$ The charging transitions sometimes occur at biases that are larger than one would expect for a $\mathrm{GaAs}-\mathrm{AlGaAs}$ heterostructure. This is likely a consequence of imperfect electrical contacts to the sample.

${ }^{15}$ A. S. Bracker, E. A. Stinaff, D. Gammon, M. E. Ware, J. G. Tischler, A. Shabaev, Al. L. Efros, D. Park, D. Gershoni, V. L. Korenev, and I. A. Merkulov, Phys. Rev. Lett. 94, 047402 (2005).

${ }^{16}$ D. Gammon, E. S. Snow, B. V. Shanabrook, D. S. Katzer, and D. Park, Phys. Rev. Lett. 76, 3005 (1996).

${ }^{17}$ A. S. Bracker, J. G. Tischler, V. L. Korenev, and D. Gammon, Phys. Status Solidi B 238, 266 (2003). 